\title{
The Effect of Neo-adjuvant Cancer Therapy on Fitness for Anaesthesia and Surgery; A Prospective Cohort Study
}

\section{Samir Rahmani ${ }^{1 *}$, Dermot Burke², Paul Jarvis ${ }^{3}$, Nicolas Orsi ${ }^{4}$, Michele Cummings $^{4}$, Shelagh Turvill ${ }^{5}$ and Simon J Howell ${ }^{2}$}

${ }^{1}$ Department of Surgery, Nevill Hall Hospital, Abergavenny, Monmouthshire, UK

${ }^{2}$ Leeds Institute of Biomedical and Clinical Sciences, University of Leeds, St. James's

University Hospital, Leeds, UK

${ }^{3}$ Faculty of Life Sciences and Education, University of South Wales, Pontypridd, UK

${ }^{4}$ Leeds Institute of Cancer and Pathology, University of Leeds, Leeds, UK

${ }^{5}$ Department of Anaesthesia, Leeds Teaching Hospitals NHS Trust, Leeds, UK

*Corresponding Author: Samir Rahmani, Nevill Hall Hospital, Department of

Surgery, Abergavenny, Monmouthshire, UK.
Received: October 12, 2020

Published: November 19, 2020

(C) All rights are reserved by Samir Rahmani., et al.

\begin{abstract}
Background: Rectal cancer treatment with neoadjuvant radiotherapy or chemoradiotherapy (NAT) prior to anaesthesia and surgery may reduce fitness and so increase perioperative risk. Understanding the mechanisms by which NAT reduces functional capacity will inform the development of preoperative risk reduction strategies such as prehabilitation. We hypothesized that the effects of NAT are mediated through the inflammatory response and changes in body composition with loss of muscle.

Objectives: The objectives of the study were to examine the associations between changes in functional reserve measured by cardiopulmonary exercise testing (CPET) and the inflammatory responses and changes in body composition produced by NAT.

Design: Prospective cohort study.

Setting: United Kingdom regional cancer centre.

Patients: Thirty-six patients (28 male) who received preoperative long course chemoradiotherapy (LCCR) $n=24$, or short course radiotherapy (SCRT) $n=12$ prior to rectal cancer surgery were studied. Patients unable to perform CPET were excluded.

Main Outcome Measures: Assessments were made before and within two weeks after NAT. Anaerobic threshold (AT) and peak oxygen consumption $\left(\mathrm{VO}_{2}\right.$ peak) were measured by CPET. Body composition was assessed by bioelectrical impedance and blood samples taken for assay of cytokines and other inflammatory markers.

Results: LCCR patients had a significant decline in $\mathrm{VO}_{2}$ peak $(\mathrm{p}=0.005)$ and reductions in several anthropometric variables $($ all $\mathrm{p}<$ 0.01 ) after NAT. SCRT patients had no significant changes in their functional capacity data but showed changes in a number of anthropometric variables (all $\mathrm{p}<0.04$ ) after NAT. Cytokines and inflammatory markers showed wide variability. There were no clinically important associations between changes in functional capacity and changes in either anthropomorphic variables or biomarkers.

Conclusion: Our data confirm the impact of NAT on fitness for surgery. They are not consistent with an association between the changes in functional capacity seen with preoperative NAT and either changes in body composition or the inflammatory response to preoperative cancer treatment.
\end{abstract}

Keywords: Neoadjuvant Therapy; Radiotherapy; Chemoradiotherapy

\section{Introduction}

Colorectal Cancer (CRC) is the second commonest cancer causing death from malignant disease in the United Kingdom
(UK). There are approximately 41,000 new cases and over 16,000 deaths each year in the UK, with an overall 5 year survival of approximately $59 \%$ [1]. Nearly $25 \%$ of colorectal tumours are rectal 
with an additional $10 \%$ in the rectosigmoid junction [2]. Although radical surgical resection of colorectal cancer is the mainstay of treatment, surgery alone has a high local recurrence rate of up to $30-50 \%$ in patients with advanced stages of rectal cancer [3]. Over the past two decades it has become clear that large rectal tumours can be down-staged with preoperative radiotherapy or chemoradiotherapy (collectively known as Neoadjuvant Therapy-NAT). In rectal cancer, the response rate after treatment with preoperative chemotherapy and radiotherapy is approximately double that after radiotherapy alone (20\% vs. $10 \%$ ) [4]. The timing of surgical resection after NAT administration is dependent on the type of the neoadjuvant treatment given and the response. It is also dependent on the size, location, extent and grade of the rectal carcinoma $[5,6]$.

There is a concern that NAT is a two-edged sword. Although enabling better treatment of the rectal cancer, NAT may increase the incidence of postoperative mortality and morbidity [7]. There are data to suggest that preoperative NAT reduces physical fitness and functional capacity and that this in turn is associated with an increased risk of perioperative complications [8-10]. The biological effects of cancer treatments have been extensively researched but the specific mechanisms by which NAT reduces functional capacity are not fully understood. Chemotherapeutic agents have been shown to reduce muscle bulk and muscle fibre size and density, effectively contributing to the sarcopenia seen in many cancer patients. The inflammatory responses to chemotherapy and chemoradiotherapy may also contribute to functional impairment [11]. The aims of the current study were:

- $\quad$ To determine if preoperative chemoradiotherapy and radiotherapy regimens produce changes in muscle bulk detectable by standard measures of body composition and anthropomorphic measurements.

- $\quad$ To determine if changes in body composition are associated with changes in functional capacity as assessed by cardiopulmonary exercise testing, so supporting the proposition that changes in muscle bulk contribute to the impact of NAT on fitness.

- To examine the association between the inflammatory and cytokine response to NAT and changes in functional capacity.

The current study was a pilot study to test the feasibility of a larger scale study of the association between changes in body composition and functional capacity, in particular the feasibility of identifying and enrolling patients in whom preoperative neoadjuvant therapy was planned and of performing assessments on these patients before and after NAT. Data from the current study would also inform sample size estimation for a larger study.

Data were also collected on quality of life outcomes. These are not included in the current publication.

\section{Methods}

Ethical approval for this study (REC reference number 09/H/1306/79) was given by the Leeds (East) Research Ethics Committee on $8^{\text {th }}$ October 2009 . Informed consent was obtained from all participants.

Patients with an advanced rectal tumour on pre-operative staging who were deemed to require NAT prior to surgery were identified and recruited from the multi-disciplinary team meeting. Patients were recruited into the study if they required combined chemotherapy and radiotherapy (LCCR) or pelvic radiotherapy alone (SCRT).

Each patient was provided with two appointments to attend the cardiac physiology department at the Leeds General Infirmary one before and one after NAT. This clinic is located at the cardiology physiology department of Leeds General Infirmary. At each visit the following assessments were made:

- Cardiopulmonary fitness (assessed with cardiopulmonary exercise testing)

- $\quad$ Body composition analysis (measured with BIA)

- $\quad$ Anthropometric measurements - body weight, height, body mass index, triceps skin folds, mid arm circumference and hand grip measurements.

Within two weeks of completion of the adjuvant pre-operative therapy, patients were invited for the second visit, to repeat the same assessments above.

\section{Cardiopulmonary exercise testing (CPET)}

Cardiopulmonary fitness was measured with the CPET equipment at the Leeds General Infirmary exercise testing laboratory. Pre-test baseline data collected included patient characteristics, cardiopulmonary history, renal function and recent medication. The CPET test equipment consisted of an electronically braked cycle ergometer, continuous 12 lead ECG monitor and a gas analyser/metabolic cart. Patients underwent CPET testing following the recommendations of the American Thoracic Society/American Association of Chest Physicians [12]. 
The anaerobic threshold (AT) was determined by dual criteria of the $\mathrm{V}$-slope method and analysis of $\mathrm{VE} / \mathrm{VCO}_{2}$ and end tidal $\mathrm{PO}_{2}$ relations. The ventilatory equivalent for carbon dioxide $\mathrm{VE} / \mathrm{VCO}_{2}$ (the ratio of expired volume to the volume of carbon dioxide excreted) was recorded at anaerobic threshold. $\mathrm{VO}_{2}$ peak was taken as the oxygen consumption ( $\mathrm{ml} \mathrm{kg}^{-1} \mathrm{~min}^{-1}$ ) at the maximum level of exercise that a subject could attain. The peak oxygen pulse, a derived measure that is a surrogate for left ventricular ejection volume and estimates oxygen delivery in millilitres per heart-beat, was also recorded.

Body composition

- Anthropometric measurements: A number of anthropometric measurements were taken including body weight $(\mathrm{kg})$, height $(\mathrm{m})$, body mass index, mid-arm circumference, triceps skin-fold, and grip strength [13].

- $\quad$ Mid-arm circumference (MAC): MAC was measured with a tape measure to the nearest $0.1 \mathrm{~cm}$. It is an indicator of bone free mid-arm muscle mass and is sensitive to muscle wasting.

- $\quad$ Triceps skin fold (TSF): The subcutaneous triceps skin folds were measured using a (Holtain Ltd, Dyfed, UK) calliper pressure $10 \mathrm{~g} \mathrm{~mm}^{-2}$, precise to $0.2 \mathrm{~mm}$. This method of nutritional screening uses a validated equation to derive a body fat percentage. This is based upon the assumption that the subcutaneous fat is a proportional reflection of the total body fat and that the sites selected for measurement represent the average fat thickness all over the body.

- Hand grip strength measurement (HG): Handgrip strength is an indicator of muscle strength and fitness. Measurements were made by using a Takei Hand Grip Dynamometer/Digital A5402 [14].

- Bioelectrical impedance Analysis (BIA): BIA is a reproducible and non-invasive method to estimate intracellular, extracellular and total body water using a $50 \mathrm{kHz}$ alternating electrical current passed between skin electrodes placed on the hands and feet [15]. Measurements in this study were performed using a HYDRA ECF/ICF (Xitron, model 4200) analyser. The mean of three consecutive measurements taken over a 30 minute period was recorded [16].

Cytokines and inflammatory markers

Blood samples were taken before the start of NAT (at the time of CPET) and after the final dose of neoadjuvant therapy for measurement of cytokines and inflammatory markers. Samples were centrifuged at $12,000 \mathrm{rpm}$ for 10 minutes at $4^{\circ} \mathrm{C}$ and plasma was isolated and stored at $-80^{\circ} \mathrm{C}$ for later analysis. Samples were defrosted and underwent batch analysis at the end of the study by fluid-phase multiplex immunoassay on a Luminex 100 cytometer (Luminex Corporation, Austin, Texas, USA) equipped with Bio-Plex software (Bio Rad, Hemel Hempstead, UK). The proinflammatory cytokines TNF-alpha and IL-1beta and the anti-inflammatory cytokines TGF-beta, IL-10 and IL1-ra were assayed [17]. In addition, a number of cytokines that have been linked to cancer progression were measured. These included IL-6, IL-12, IL-18, macrophage migration inhibitory factor (MIF), IP-10 (also known as C-X-C motif chemokine 10 or CXCL10), RANTES (also known as Chemokine (C-C motif) ligand or CCL5)) [18-22]. Serum albumin, which has been linked to colorectal cancer progression as a marker of inflammation [23], was also assayed using a bromocresyl green-based assay (Randox Laboratories, Co. Antrim, UK) and absorbance read at $570 \mathrm{~nm}$ (Opsys MR 15, Dynex Technologies, Worthing, UK). Creactive protein was analysed by enhanced latex-turbidometric immunoassay (Thermo Electron Corporation, Alpha Laboratories, Hampshire, UK) on an automated clinical autoanalyser (COBAS MIRA, Roche, Basel, Switzerland). All analyses were performed in duplicate.

\section{Neoadjuvant therapy}

It is established that combination regimens provide improved efficacy and prolonged progression-free survival in patients with colorectal cancer [24]. Over the past two decades it has become clear that large rectal tumours can be down staged with preoperative treatment.

- $\quad$ Short course radiotherapy (SCRT): Preoperative highdose short-course radiotherapy for five days has been shown to reduce the risk of local recurrence and to improve survival, after curative surgery to rectal cancer [25].

- Long course chemoradiotherapy (LCCR): In locally advanced and initially unresectable rectal cancers (T3 or greater, node positive), combined chemoradiotherapy may downstage the tumour and allow potentially curative surgery [26]. Pre-operative 5-fluorouracil (5-FU) administration increases the bowel sensitivity to radiotherapy. This combined therapy improves local control, distant spread and survival [27].

\section{Statistics}

As this was a pilot study to test the feasibility of a larger study no power calculation was performed. Sample size was determined by logistical considerations and by the number of patients eligible 
for recruitment during the study period. Statistical analyses as described below were planned and performed to identify any detectable effects within the constraints of this small study.

Descriptive statistics are given as median and range. Percentages are given for categorical data where appropriate. Non-parametric tests were used to compare paired related samples (Wilcoxon Rank Sum Test). Associations between changes in anthropomorphic and function capacity were examined using Spearman rank correlation tests. As this was a pilot study no corrections for multiple comparisons were made (so risking Type I error but avoiding Type II error) in order to increase the likelihood of identifying potentially significant findings to be explored further in a larger study. Analyses were performed using Intercooled Stata version 13. A p-value $<0.05$ was considered statistically significant.

\section{Results}

Thirty-six patients were recruited to the study; twenty-four chemoradiotherapy patients and twelve who received radiotherapy alone.

\section{Chemoradiotherapy group (LCCR)}

Twenty-four patients (18 male) received combined chemoradiotherapy. The median (range) age of this group was 61 (23.7 80.8) years. Eleven patients had intravenous chemotherapy with pelvic radiotherapy and 13 patients had oral chemotherapy with pelvic radiotherapy. The length of LCCR in this group was 39 (25 - 92) days. The interval between finish of the NAT and the day of surgery was $36(14-65)$ days.

Functional capacity and anthropomorphic variables before and after LCCR are reported in table 1 . There was a significant decrease in $\mathrm{VO}_{2}$ peak after LCCR. Anaerobic threshold was reduced in most of the patients but the difference did not reach statistical significance. There was a significant increase in the $\mathrm{VE} / \mathrm{VCO}_{2}$ which is a measure of the ventilation required to eliminate carbon dioxide and so is a measure of respiratory efficiency. There was no significant change in oxygen pulse, which reflects oxygen uptake per heartbeat and is a surrogate for cardiac output. There was no significant change in the body weight and body mass index post LCCR. There was a significant reduction in grip strength following LCCR. Mid-arm circumference which is a measure of overall nutritional status and triceps skin fold which reflects fat thickness were reduced. Other anthropomorphic variables were unchanged. There were no significant associations between changes in anaerobic threshold and $\mathrm{VO}_{2}$ peak and changes in anthropomorphic variables (Table 2).

There was no significant change in albumin following LCCR. There was a statistically significant reduction in C-reactive protein following LCCR. Cytokine values showed great variation between subjects as indicated by the wide interquartile ranges in table 2 . There was a statistically significant reduction in IL1-ra following LCCR but no significant changes in cytokine concentrations (Table 3). There were no significant correlations between changes in cytokines or inflammatory markers and objective measures of functional capacity (Table 4).

\begin{tabular}{|l|c|c|c|c|c|c|c|c|}
\hline & \multicolumn{4}{|c|}{ Chemoradiotherapy N=24 } & \multicolumn{3}{c|}{ Radiotherapy N=12 } \\
\cline { 2 - 9 } & Pre APT & Post APT & Change & p & Pre APT & Post APT & Change & p \\
\hline $\begin{array}{l}\text { Functional Reserve } \\
\text { Indices }\end{array}$ & & & & & & & \\
\hline Peak Power & 102.0 & 112.5 & -2.0 & 0.843 & 89.5 & 81.0 & -5.0 & 0.054 \\
(Watts) & $81.3-139.8$ & $68.8-154.8$ & $-15.0-13.0$ & & $62.8-133.0$ & $65.3-126.5$ & $-15.3-2.8$ & \\
\hline Anaerobic Threshold & 13.5 & 12.3 & -1.0 & 0.065 & 11.6 & 11.0 & -0.8 & 0.239 \\
$\mathrm{ml} \mathrm{kg}^{-1} \mathrm{~min}^{-1}$ & $12.5-16.2$ & $10.2-13.7$ & $-4.1-0.5$ & & $9.9-14.5$ & $10.4-13.2$ & $-3.3-0.5$ & \\
\hline V02peak & 19.4 & 17.1 & -1.0 & 0.005 & 18.4 & 16.3 & -0.4 & 0.398 \\
$\mathrm{ml} \mathrm{kg}^{-1} \mathrm{~min}^{-1}$ & $15.6-22.5$ & $14.6-20.2$ & $-2.8-0.03$ & & $12.9-22.1$ & $12.3-20.3$ & $-2.6-1.2$ & \\
\hline VE/VC02 at AT & 29.5 & 32.5 & 2.0 & $<0.001$ & 33.0 & 34.5 & 1.5 & 0.180 \\
& $28.0-34.0$ & $28.3-37.0$ & $1.0-3.0$ & & $30.0-42.0$ & $30.2-42.3$ & $-1.5-4.0$ & \\
\hline Peak 02 Pulse & 9.0 & 9.0 & -0.5 & 0.498 & 8.5 & 8.5 & -0.5 & 0.222 \\
(ml beat $^{-1}$ ) & $6.3-10.8$ & $6.3-11.0$ & $-1.0-1.0$ & & $6.3-9.8$ & $6.3-9.8$ & $-1.0-0.0$ & \\
\hline
\end{tabular}




\begin{tabular}{|c|c|c|c|c|c|c|c|c|}
\hline $\begin{array}{l}\text { Anthropomorphic } \\
\text { Indices }\end{array}$ & & & & & & & & \\
\hline $\begin{array}{l}\text { Weight } \\
(\mathrm{kg})\end{array}$ & $\begin{array}{c}68.6 \\
59.6-79.5\end{array}$ & $\begin{array}{c}66.5 \\
57.9-80.4\end{array}$ & $\begin{array}{c}-0.7 \\
-2.7-1.1\end{array}$ & 0.271 & $\begin{array}{c}70.6 \\
62.5-90.8\end{array}$ & $\begin{array}{c}71.5 \\
61.6-90.4\end{array}$ & $\begin{array}{c}-0.3 \\
-0.6-1.0\end{array}$ & 0.937 \\
\hline $\begin{array}{l}\text { BMI } \\
\left(\mathrm{kg} \mathrm{m}^{-2}\right)\end{array}$ & $\begin{array}{c}26.0 \\
21.2-28.3\end{array}$ & $\begin{array}{c}25.4 \\
21.5-28.4\end{array}$ & $\begin{array}{c}-0.1 \\
-1.0-0.8\end{array}$ & 0.700 & $\begin{array}{c}25.8 \\
24.0-30.1\end{array}$ & $\begin{array}{c}25.5 \\
23.8-29.6\end{array}$ & $\begin{array}{c}-0.2 \\
-0.4-0.2\end{array}$ & 0.209 \\
\hline $\begin{array}{l}\text { Triceps skin fold } \\
(\mathrm{mm})\end{array}$ & $\begin{array}{c}13.0 \\
7.9-16.3\end{array}$ & $\begin{array}{c}10.5 \\
8.0-13.9\end{array}$ & $\begin{array}{c}-1.1 \\
-2.0--0.1\end{array}$ & 0.007 & $\begin{array}{c}12.4 \\
9.0-22.8\end{array}$ & $\begin{array}{c}9.2 \\
7.7-18.5\end{array}$ & $\begin{array}{c}-2.0 \\
-3.0--0.4\end{array}$ & 0.013 \\
\hline $\begin{array}{l}\text { Mid-arm circumfer- } \\
\text { ence } \\
(\mathrm{cm})\end{array}$ & $\begin{array}{c}28.5 \\
24.6-30.7\end{array}$ & $\begin{array}{c}27.6 \\
23.9-30.0\end{array}$ & $\begin{array}{c}-1.0 \\
-2.8-0.0\end{array}$ & 0.006 & $\begin{array}{c}29.5 \\
26.9-31.8\end{array}$ & $\begin{array}{c}28.5 \\
26.0-31.0\end{array}$ & $\begin{array}{c}-0.9 \\
-2.0-0.0\end{array}$ & 0.013 \\
\hline $\begin{array}{l}\text { Total Body Water } \\
\text { (litre) }\end{array}$ & $\begin{array}{c}34.8 \\
26.5-40.8\end{array}$ & $\begin{array}{c}32.8 \\
25.2-41.1\end{array}$ & $\begin{array}{c}0.3 \\
-1.2-1.0\end{array}$ & 0.841 & $\begin{array}{c}33.6 \\
30.4-43.7\end{array}$ & $\begin{array}{c}33.6 \\
31.3-42.0\end{array}$ & $\begin{array}{c}-0.9 \\
-1.8-1.0 \\
\end{array}$ & 0.433 \\
\hline $\begin{array}{l}\text { Intracellular Water } \\
\text { (litre) }\end{array}$ & $\begin{array}{c}19.1 \\
13.1-21.8\end{array}$ & $\begin{array}{c}17.2 \\
12.8-22.0\end{array}$ & $\begin{array}{c}0.0 \\
-1.0-0.4\end{array}$ & 0.391 & $\begin{array}{c}18.0 \\
15.7-23.1\end{array}$ & $\begin{array}{c}17.7 \\
15.8-21.3\end{array}$ & $\begin{array}{c}-0.7 \\
-2.0-0.8\end{array}$ & 0.327 \\
\hline $\begin{array}{l}\text { Extracellular Water } \\
\text { (litre) }\end{array}$ & $\begin{array}{c}15.8 \\
13.2-18.6\end{array}$ & $\begin{array}{c}15.9 \\
12.1-18.6\end{array}$ & $\begin{array}{c}0.4 \\
-0.5-0.9 \\
\end{array}$ & 0.415 & $\begin{array}{c}16.7 \\
14.6-19.3\end{array}$ & $\begin{array}{c}16.9 \\
14.5-19.2\end{array}$ & $\begin{array}{c}0.0 \\
-0.5-0.6\end{array}$ & 0.695 \\
\hline $\begin{array}{l}\text { Fat free mass } \\
(\mathrm{kg})\end{array}$ & $\begin{array}{c}44.8 \\
34.1-52.9\end{array}$ & $\begin{array}{c}42.4 \\
33.7-53.5\end{array}$ & $\begin{array}{c}-0.2 \\
-1.6-1.3\end{array}$ & 0.864 & $\begin{array}{c}48.2 \\
42.4-56.4\end{array}$ & $\begin{array}{c}44.2 \\
40.5-55.1\end{array}$ & $\begin{array}{c}-1.6 \\
-4.1--0.7\end{array}$ & 0.034 \\
\hline $\begin{array}{l}\text { Grip Strength } \\
\text { (kg) }\end{array}$ & $\begin{array}{c}31.2 \\
24.8-43.3\end{array}$ & $\begin{array}{c}30.1 \\
24.3-42.4\end{array}$ & $\begin{array}{c}-2.2 \\
-4.3-1.1\end{array}$ & 0.010 & $\begin{array}{c}34.5 \\
29.8-38.5\end{array}$ & $\begin{array}{c}33.7 \\
30.0-36.8\end{array}$ & $\begin{array}{c}-1.3 \\
-3.2-0.8\end{array}$ & 0.158 \\
\hline
\end{tabular}

Table 1: Cardiopulmonary variables and anthropomorphic indices before and after neoadjuvant therapy. Values are given as median and interquartile range.

\begin{tabular}{|c|c|c|c|c|c|c|c|c|c|c|}
\hline \multicolumn{11}{|c|}{ Chemoradiotherapy N=24 } \\
\hline \multirow{2}{*}{\multicolumn{2}{|c|}{ Weight }} & \multicolumn{9}{|c|}{ Change in } \\
\hline & & BMI & $\begin{array}{l}\text { Triceps } \\
\text { skin fold }\end{array}$ & $\begin{array}{l}\text { Mid-arm } \\
\text { circumfer- } \\
\text { ence }\end{array}$ & $\begin{array}{c}\text { Grip } \\
\text { Strength }\end{array}$ & $\begin{array}{c}\text { Fat free } \\
\text { mass }\end{array}$ & $\begin{array}{l}\text { Intracel- } \\
\text { lular } \\
\text { Water }\end{array}$ & $\begin{array}{l}\text { Extra- } \\
\text { cellular } \\
\text { Water }\end{array}$ & $\begin{array}{l}\text { Total } \\
\text { Body } \\
\text { Water }\end{array}$ & \\
\hline \multirow{2}{*}{$\begin{array}{l}\text { Change } \\
\text { in }\end{array}$} & $\begin{array}{l}\text { Anaerobic } \\
\text { Threshold }\end{array}$ & $\begin{array}{l}-0.182 \\
(0.395)\end{array}$ & $\begin{array}{l}-0.025 \\
(0.908)\end{array}$ & $\begin{array}{l}-0.151 \\
(0.482)\end{array}$ & $\begin{array}{l}-0.091 \\
(0.673)\end{array}$ & $\begin{array}{c}0.139 \\
(0.517)\end{array}$ & $\begin{array}{c}0.295 \\
(0.161)\end{array}$ & $\begin{array}{c}0.268 \\
(0.206)\end{array}$ & $\begin{array}{c}0.100 \\
(0.640)\end{array}$ & $\begin{array}{r}0.299 \\
(0.156) \\
\end{array}$ \\
\hline & V02peak & $\begin{array}{c}-0.44 \\
(0.837)\end{array}$ & $\begin{array}{c}0.201 \\
(0.347)\end{array}$ & $\begin{array}{c}0.241 \\
(0.257)\end{array}$ & $\begin{array}{c}0.257 \\
(0.225)\end{array}$ & $\begin{array}{c}-0.22 \\
(0.295)\end{array}$ & $\begin{array}{c}0.083 \\
(0.701)\end{array}$ & $\begin{array}{c}0.166 \\
(0.438)\end{array}$ & $\begin{array}{c}0.092 \\
(0.670)\end{array}$ & $\begin{array}{r}0.134 \\
(0.534) \\
\end{array}$ \\
\hline \multicolumn{11}{|c|}{ Radiotherapy N=12 } \\
\hline \multirow{2}{*}{\multicolumn{2}{|c|}{$\begin{array}{l}\text { Weight } \\
\mathrm{kg}\end{array}$}} & \multicolumn{9}{|c|}{ Change in } \\
\hline & & $\begin{array}{c}\text { BMI } \\
\text { Kg m-2 }\end{array}$ & $\begin{array}{l}\text { Triceps } \\
\text { skin fold } \\
\text { cm }\end{array}$ & $\begin{array}{l}\text { Mid-arm } \\
\text { circumfer- } \\
\text { ence cm }\end{array}$ & $\begin{array}{l}\text { Grip } \\
\text { Strength } \\
\text { kg }\end{array}$ & $\begin{array}{l}\text { Fat free } \\
\text { mass kg }\end{array}$ & $\begin{array}{c}\text { Intracel- } \\
\text { lular } \\
\text { Water }\end{array}$ & $\begin{array}{l}\text { Extra- } \\
\text { cellular } \\
\text { Water }\end{array}$ & $\begin{array}{l}\text { Total } \\
\text { Body } \\
\text { Water }\end{array}$ & \\
\hline \multirow{2}{*}{$\begin{array}{l}\text { Change } \\
\text { in }\end{array}$} & $\begin{array}{l}\text { Anaerobic } \\
\text { Threshold } \\
\left(\mathrm{ml} \mathrm{kg}^{-1} \mathrm{~min}^{-1}\right)\end{array}$ & $\begin{array}{c}0.615 \\
(0.033)\end{array}$ & $\begin{array}{l}-0.420 \\
(0.175)\end{array}$ & $\begin{array}{l}-0.377 \\
(0.226)\end{array}$ & $\begin{array}{c}0.523 \\
(0.081)\end{array}$ & $\begin{array}{c}0.713 \\
(0.009)\end{array}$ & $\begin{array}{c}0.021 \\
(0.948)\end{array}$ & $\begin{array}{c}0.524 \\
(0.080)\end{array}$ & $\begin{array}{l}-0.126 \\
(0.697)\end{array}$ & $\begin{array}{r}0.531 \\
(0.075) \\
\end{array}$ \\
\hline & $\begin{array}{l}\text { V02peak } \\
\left(\mathrm{ml} \mathrm{kg}^{-1} \mathrm{~min}^{-1}\right)\end{array}$ & $\begin{array}{l}-0.354 \\
(0.259)\end{array}$ & $\begin{array}{l}-0.025 \\
(0.940)\end{array}$ & $\begin{array}{r}0.269 \\
(0.399) \\
\end{array}$ & $\begin{array}{c}0.071 \\
(0.827)\end{array}$ & $\begin{array}{c}0.455 \\
(0.137)\end{array}$ & $\begin{array}{l}-0.214 \\
(0.505)\end{array}$ & $\begin{array}{c}0.567 \\
(0.054)\end{array}$ & $\begin{array}{l}-0.249 \\
(0.436)\end{array}$ & $\begin{array}{r}0.539 \\
(0.070) \\
\end{array}$ \\
\hline
\end{tabular}

Table 2: Correlation coefficients (and p-values in parenthesis) for changes in cardiopulmonary and anthropomorphic variables following chemoradiotherapy or radiotherapy. 


\begin{tabular}{|c|c|c|c|c|c|c|c|c|}
\hline & \multicolumn{4}{|c|}{ Chemoradiotherapy $\mathrm{N}=\mathbf{2 0}$} & \multicolumn{4}{|c|}{ Radiotherapy $\mathrm{N}=12$} \\
\hline & Pre APT & Post APT & Change & $\mathbf{p}$ & Pre APT & Post APT & Change & $\mathbf{p}$ \\
\hline $\begin{array}{l}\text { IL1-beta } \\
\left(\mathrm{pg} \mathrm{ml}^{-1}\right)\end{array}$ & $\begin{array}{c}0.68 \\
0.59-1.12 \\
\end{array}$ & $\begin{array}{c}0.53 \\
0.48-1.00 \\
\end{array}$ & $\begin{array}{c}-0.06 \\
-0.21--0.01 \\
\end{array}$ & 0.076 & $\begin{array}{c}0.59 \\
0.49-1.22 \\
\end{array}$ & $\begin{array}{c}0.53 \\
0.49-0.77 \\
\end{array}$ & $\begin{array}{c}-0.02 \\
-0.28-0.00\end{array}$ & 0.069 \\
\hline $\begin{array}{l}\text { IL1-ra } \\
\left(\mathrm{pg} \mathrm{ml}{ }^{-1}\right)\end{array}$ & $\begin{array}{c}18.19 \\
8.90-68.76\end{array}$ & $\begin{array}{c}11.01 \\
6.25-81.57\end{array}$ & $\begin{array}{c}-5.17 \\
-18.16--0.28\end{array}$ & 0.036 & $\begin{array}{c}10.11 \\
6.25-22.69\end{array}$ & $\begin{array}{c}8.60 \\
7.42-13.94\end{array}$ & $\begin{array}{c}-1.46 \\
-3.21-1.77\end{array}$ & 0.388 \\
\hline $\begin{array}{l}\text { IL6 } \\
\left(\mathrm{pg} \mathrm{ml}^{-1}\right)\end{array}$ & $\begin{array}{c}9.40 \\
4.14-74.34 \\
\end{array}$ & $\begin{array}{c}8.62 \\
3.43-29.64 \\
\end{array}$ & $\begin{array}{c}-1.41 \\
-8.66-0.56\end{array}$ & 0.093 & $\begin{array}{c}5.96 \\
5.06-9.06 \\
\end{array}$ & $\begin{array}{c}6.98 \\
5.53-20.45 \\
\end{array}$ & $\begin{array}{c}2.75 \\
-0.06-10.32 \\
\end{array}$ & 0.034 \\
\hline $\begin{array}{l}\text { IL10 } \\
\left(\mathrm{pg} \mathrm{ml}{ }^{-1}\right)\end{array}$ & $\begin{array}{c}2.91 \\
1.33-39.90\end{array}$ & $\begin{array}{c}2.16 \\
1.49-55.20\end{array}$ & $\begin{array}{c}-0.21 \\
-5.02-0.40\end{array}$ & 0.370 & $\begin{array}{c}1.55 \\
1.07-2.33\end{array}$ & $\begin{array}{c}1.93 \\
1.33-2.84 \\
\end{array}$ & $\begin{array}{c}0.42 \\
-0.16-1.45\end{array}$ & 0.213 \\
\hline $\begin{array}{l}\text { Chemokine } \\
\text { (C-C motif) } \\
\text { ligand } 5 \\
\text { (CCL5) or } \\
\text { RANTES } \\
\left.(\mathrm{pg} \mathrm{ml})^{-1}\right)\end{array}$ & $\begin{array}{c}1594.5 \\
1203.7-2025.8\end{array}$ & $\begin{array}{c}1506.2 \\
1065.1-1731.6\end{array}$ & $\begin{array}{c}-178.5 \\
-530.9-232.6\end{array}$ & 0.232 & $\begin{array}{c}1353.0 \\
677.0-1591.1\end{array}$ & $\begin{array}{c}1390.6 \\
1017.5-1921.7\end{array}$ & $\begin{array}{c}-47.2 \\
-477.7-799.0\end{array}$ & 1.000 \\
\hline $\begin{array}{l}\text { TNF-alpha } \\
\left(\mathrm{pg} \mathrm{ml}^{-1}\right)\end{array}$ & $\begin{array}{c}7.27 \\
4.88-15.31 \\
\end{array}$ & $\begin{array}{c}3.94 \\
2.08-17.78 \\
\end{array}$ & $\begin{array}{c}-2.80 \\
-5.71--0.24\end{array}$ & 0.070 & $\begin{array}{c}4.65 \\
2.66-7.27 \\
\end{array}$ & $\begin{array}{c}3.47 \\
3.00-5.59 \\
\end{array}$ & $\begin{array}{c}-0.71 \\
-2.31-0.35\end{array}$ & 0.074 \\
\hline $\begin{array}{l}\text { C-reactive } \\
\text { protein } \\
\mathrm{mg} \mathrm{dl}^{-1}\end{array}$ & $\begin{array}{c}0.43 \\
0.22-0.76\end{array}$ & $\begin{array}{c}0.23 \\
0.05-0.63\end{array}$ & $\begin{array}{c}-0.11 \\
-0.43-0.02\end{array}$ & 0.033 & $\begin{array}{c}0.13 \\
0.10-0.74\end{array}$ & $\begin{array}{c}0.36 \\
0.21-0.56\end{array}$ & $\begin{array}{c}0.15 \\
0.02-0.35\end{array}$ & 0.084 \\
\hline $\begin{array}{l}\text { Albumin } \\
\text { gm dl}^{-1}\end{array}$ & $\begin{array}{c}3.89 \\
3.51-4.20 \\
\end{array}$ & $\begin{array}{c}3.77 \\
3.44-4.00\end{array}$ & $\begin{array}{c}-0.14 \\
-0.48-0.11\end{array}$ & 0.121 & $\begin{array}{c}4.00 \\
3.71-4.18\end{array}$ & $\begin{array}{c}3.91 \\
3.55-4.03\end{array}$ & $\begin{array}{c}0.00 \\
-0.31-0.22\end{array}$ & 0.753 \\
\hline
\end{tabular}

Table 3: Cytokines and inflammatory markers before and after neoadjuvant therapy. Values are given as median and interquartile range. 


\begin{tabular}{|c|c|c|c|c|c|c|c|c|c|c|c|c|}
\hline \multicolumn{13}{|c|}{ Chemoradiotherapy $\mathrm{N}=20$} \\
\hline \multirow{2}{*}{\multicolumn{2}{|c|}{ IL1-beta }} & \multicolumn{11}{|c|}{ Change in } \\
\hline & & IL1-ra & IL6 & IL10 & IP10 & CCL5 & $\begin{array}{l}\text { TNF- } \\
\text { alpha }\end{array}$ & IL18 & MIF & CRP & $\begin{array}{l}\text { Albu- } \\
\text { min }\end{array}$ & \\
\hline \multirow{2}{*}{$\begin{array}{c}\text { Change } \\
\text { in }\end{array}$} & $\begin{array}{l}\text { An- } \\
\text { aerobic } \\
\text { Thresh- } \\
\text { old }\end{array}$ & $\begin{array}{c}0.267 \\
(0.203)\end{array}$ & $\begin{array}{c}0.290 \\
(0.214)\end{array}$ & $\begin{array}{c}0.372 \\
(0.163)\end{array}$ & $\begin{array}{c}0.305 \\
(0.284)\end{array}$ & $\begin{array}{l}-0.141 \\
(0.559)\end{array}$ & $\begin{array}{l}-0.105 \\
(0.650)\end{array}$ & $\begin{array}{c}0.401 \\
(0.080)\end{array}$ & $\begin{array}{r}0.101 \\
(0.650)\end{array}$ & $\begin{array}{l}-0.171 \\
(0.470)\end{array}$ & $\begin{array}{c}0.313 \\
(0.179)\end{array}$ & $\begin{array}{c}-0.043 \\
(0.857)\end{array}$ \\
\hline & $\mathrm{VO}_{2}$ peak & $\begin{array}{r}-0.385 \\
(0.094)\end{array}$ & $\begin{array}{c}-0.049 \\
(0.838)\end{array}$ & $\begin{array}{l}-0.087 \\
(0.714) \\
\end{array}$ & $\begin{array}{r}0.099 \\
(0.679) \\
\end{array}$ & $\begin{array}{c}0.024 \\
(0.920) \\
\end{array}$ & $\begin{array}{r}0.249 \\
(0.347) \\
\end{array}$ & $\begin{array}{r}-0.272 \\
(0.134) \\
\end{array}$ & $\begin{array}{r}0.089 \\
(0.673) \\
\end{array}$ & $\begin{array}{r}-0.053 \\
(0.823) \\
\end{array}$ & $\begin{array}{c}0.185 \\
(0.435)\end{array}$ & $\begin{array}{r}0.158 \\
(0.507) \\
\end{array}$ \\
\hline \multicolumn{13}{|c|}{ Radiotherapy N=12 } \\
\hline \multirow{2}{*}{\multicolumn{2}{|c|}{ IL1-beta }} & \multicolumn{11}{|c|}{ Change in } \\
\hline & & IL1-ra & IL6 & IL10 & IP10 & CCL5 & $\begin{array}{l}\text { TNF- } \\
\text { alpha }\end{array}$ & IL18 & MIF & CRP & $\begin{array}{l}\text { Albu- } \\
\text { min }\end{array}$ & \\
\hline \multirow{2}{*}{$\begin{array}{l}\text { Change } \\
\text { in }\end{array}$} & $\begin{array}{l}\text { An- } \\
\text { aerobic } \\
\text { Thresh- } \\
\text { old }\end{array}$ & $\begin{array}{c}0.164 \\
(0.611)\end{array}$ & $\begin{array}{c}0.277 \\
(0.384)\end{array}$ & $\begin{array}{r}-0.154 \\
(0.633)\end{array}$ & $\begin{array}{c}0.236 \\
(0.484)\end{array}$ & $\begin{array}{c}-.035 \\
(0.914)\end{array}$ & $\begin{array}{c}0.264 \\
(0.433)\end{array}$ & $\begin{array}{r}0.070 \\
(0.829)\end{array}$ & $\begin{array}{l}-0.455 \\
(0.160)\end{array}$ & $\begin{array}{c}0.105 \\
(0.746)\end{array}$ & $\begin{array}{l}-0.336 \\
(0.286)\end{array}$ & $\begin{array}{c}0.623 \\
(0.030)\end{array}$ \\
\hline & $\mathrm{VO}_{2}$ peak & $\begin{array}{c}0.039 \\
(0.904)\end{array}$ & $\begin{array}{c}0.267 \\
(0.402)\end{array}$ & $\begin{array}{l}-0.102 \\
(0.753)\end{array}$ & $\begin{array}{l}-0.114 \\
(0.739)\end{array}$ & $\begin{array}{l}-0.102 \\
(0.753)\end{array}$ & $\begin{array}{c}0.282 \\
(0.400)\end{array}$ & $\begin{array}{l}-0.112 \\
(0.728)\end{array}$ & $\begin{array}{l}-0.027 \\
(0.936)\end{array}$ & $\begin{array}{c}0.172 \\
(0.594)\end{array}$ & $\begin{array}{l}-0.329 \\
(0.296)\end{array}$ & $\begin{array}{c}0.316 \\
(0.317)\end{array}$ \\
\hline
\end{tabular}

Table 4: Correlation coefficients (and p-values in parenthesis) for changes in cytokines and inflammatory markers and functional capacity following chemoradiotherapy or radiotherapy.

\section{Radiotherapy group (SCRT)}

Twelve patients (10 male) had only pelvic radiotherapy. The median (range) age in this group was 71.8 (55.3 - 88.4) years, and the length of adjuvant pre-operative treatment radiotherapy was 6 ( 4 - 7) days. The interval between finish of the SCRT and the day of surgery was 13.5 (8 - 58) days.

In this group, no significant changes were found in the CPET measures of functional capacity variables following SCRT. As with the patients who received LCCR, there was a significant decrease in triceps skin fold and mid arm circumference following neoadjuvant therapy. Fat free mass ( $p=0.034$ ) was also reduced significantly post SCRT. In contrast to patients receiving LCCR, there was no change in grip strength in patients who received SCRT. There was no significant change in other anthropomorphic variables (Table 1). Significant correlations were noted between change in grip strength and change in anaerobic threshold and between change in weight and change in anaerobic threshold (Table 2). No other correlations between changes in functional capacity and anthropomorphic variables achieved significance at the $\mathrm{p}<0.05$ level.
There was a statistically significant increase in IL-6 following SCRT. There were no other statistically significant changes in inflammatory markers or cytokines following SCRT (Table 3). There was a significant association between the change albumin concentration and change in anaerobic threshold following SCRT. No other significant correlations between changes in cytokines or inflammatory markers and objective measures of functional capacity were identified (Table 4).

\section{Discussion and Conclusion}

This study examined changes in cardiopulmonary functional capacity and anthropomorphic variables in patients with rectal cancer who received neoadjuvant treatment. We found a significant deterioration in cardiopulmonary reserve following long course chemoradiotherapy. This was manifest as a reduction in $\mathrm{VO}_{2}$ peak. There was no statistically significant reduction in functional reserve in patients who underwent short course radiotherapy. Chemoradiotherapy was associated with significant reductions in triceps skin fold, mid-arm circumference and grip strength. The first two are indicators of nutritional status and body fat percent- 
age. The last is an indicator of muscle strength. Short course radiotherapy was associated with a decrease in triceps skin fold and mid-arm circumference. No change was seen in grip strength. The impact of LCCR on functional capacity may reflect the longer duration of treatment (a median of 39 days as compared with 6 days in the case of SCRT) and the systemic administration of 5-fluorouracil in LCCR. Long course chemoradiotherapy has been shown to be associated with reduced energy intake and weight loss [28]. These data suggest that the loss of appetite associated with short course treatment has some nutritional impact. Changes in functional capacity and anthropomorphic measures varied between patients. The decrease in functional capacity seen in LCCR patients was not associated with changes in anthropomorphic measures or grip strength suggesting that the change in functional capacity could not be attributed to a loss of muscle bulk. The lack of any consistent associations between measures of functional capacity and body composition suggested to us that a larger study would be unlikely to demonstrate clinically important effects.

Both radiotherapy and chemoradiotherapy are associated with an inflammatory response which may produce systemic symptoms including fatigue $[11,29]$. We measured C-reactive protein and serum albumin before and after NAT as non-specific markers of inflammation. We also measured a panel of cytokines that included both markers of inflammation and cytokines specifically associated with cancer progression. The data showed a wide variation in inflammatory and cytokine responses between patients. We found statistically significant reductions in IL1-ra and C-reactive protein following LCCR. These reductions are not consistent with the hypothesis that an inflammatory response to NAT reduces functional capacity. Both IL1-ra and C-reactive protein would be expected to increase in systemic inflammation. We did find a significant increase in IL-6 following NAT in the SCRT group which would be consistent with an inflammatory response. However, there was no change in cytokines or inflammatory markers consistent with systemic inflammation following NAT in either group. As a total of 22 comparisons were made (11 in each group) it is likely that the statistically significant changes which we did observe were the result of type 1 error. Whilst we found a significant correlation between the change in serum albumin and the change in anaerobic threshold following SCRT this was again an isolated association. There were no other associations between changes in inflammatory markers and cytokines and changes in functional capacity.
These results suggest that a larger study would be unlikely to demonstrate clinically important associations between the inflammatory response to NAT and the changes in functional capacity seen with preoperative adjuvant treatment.

The measures of functional capacity and the anthropomorphic measurements used in this study have direct clinical relevance. The association between reduced functional capacity as measured by cardiopulmonary exercise testing and an increased risk of death or major complications following surgery is well established [9]. Patients with reduced functional capacity have been shown to be more likely to suffer major perioperative or postoperative complications [30,31]. Our data showed a significant reduction in the cardiac reserve, represented by $\mathrm{VO}_{2}$ peak, of those patients exposed to chemoradiotherapy. This decrease in $\mathrm{VO}_{2}$ peak is associated with an increased risk of perioperative morbidity.

Body composition refers to hydration, nutrition, wasting, bone mineral content and total body fat [31]. Triceps skin fold (TSF) and mid-arm circumference (MAC) were decreased in both groups after neo-neoadjuvant therapy. In addition, FFM was reduced in the radiotherapy only group. These changes may reflect impaired nutrition and increased wasting after neoadjuvant treatment. The incidence of malnutrition in patients with cancer ranges from 30 to $60 \%[33,34]$ Chemotherapy can affect intracellular protein synthesis, which in turn leads to deterioration in the patient's overall nutritional status [35]. It also has indirect systemic effects including nausea, vomiting, anorexia, and mucositis. Patients submitted to radiotherapy of the gastrointestinal tract are also at higher risk of malnutrition as it can cause mucositis, nausea, vomiting, diarrhoea and anorexia [36,37] Subclinical inflammation associated with NAT may lead to an increased risk of protein energy malnutrition [29]. Anti-cancer treatment may increase muscle atrophy and muscle wasting and may be mediated through activation of the NF- $\kappa B$ signalling pathway [38]. These body composition changes may be associated with an increased risk of postoperative morbidity [39].

In a study that mirrors our work in rectal cancer, Jack and colleagues studied patients with oesophageal cancer. They found that neoadjuvant chemotherapy significantly reduced physical fitness and one-year-survival in the cohort of patients undergoing surgical resection, following NAT exposure [10].

We demonstrated a decrease in cardiopulmonary reserve with LCCR and changes in anthropomorphic variables with both LCCR

Citation: Samir Rahmani., et al. "The Effect of Neo-adjuvant Cancer Therapy on Fitness for Anaesthesia and Surgery; A Prospective Cohort Study". Acta Scientific Medical Sciences 4.12 (2020): 53-63. 
and SCRT. However, we were unable to demonstrate significant associations between changes in in functional reserve and changes in either anthropomorphic variables or the inflammatory response that would support a causal relationship. There were associations between changes in grip strength, weight and anaerobic threshold in patients who underwent SCRT but there were no related associations between other indices of body composition and functional capacity in this group to support causality and it seems this isolated positive result represents type one statistical error. This is a small pilot study which may have been underpowered to detect subtle effects, especially in the SCRT group.

However, we were able to demonstrate an effect of LCCR on functional capacity and effects of both LCCR and SCRT on body composition. Whilst we found that a larger scale study would be feasible there was no suggestion of causal associations that would be confirmed by a larger study. This pilot work suggests that a larger study of the association between changes in functional capacity, body composition and the inflammatory response to NAT would be unlikely to demonstrate clinically important effects. Future work should explore other possible causes of the decrease in functional capacity seen with NAT. Possible mechanisms include interference with oxidative phosphorylation in muscle, reduced substrate delivery to muscle due to microvascular changes and direct effects on the heart and lungs [40]. Our study suggested a significant increase in the $\mathrm{VE} / \mathrm{VCO}_{2}$ post chemoradiotherapy. This is an objective measurement of the pulmonary dead space and strongly associated with poor ventilatory function, pulmonary arterial hypertension and cardiac failure [41]. Chemotherapy agents, such as 5-Fluorouracil (5FU), are known to cause coronary endothelium toxicity, myocardium hypoxia as well as toxic myocarditis $[42,43]$. These systemic agents cause microvascular damage as well as tissue hypoxia at cellular level leading to an end-organ mal-function or dysfunction. These mechanisms are areas for possible future research.

\section{Acknowledgments}

We are grateful for the technical support of Wanda MacDonald (Cardiology nurse practitioner and CPET specialist).

\section{Financial Support}

This work was supported by a grant from Yorkshire Cancer Research.

\section{Conflicts of Interest}

SJH has received honoraria from CSL Behring. No other conflicts of interest.

\section{Presentation}

The protocol and preliminary data from this project have been presented at meetings of BAPEN (British Association for Parenteral and Enteral Nutrition).

\section{Bibliography}

1. http://www.cancerresearchuk.org/health-professional/cancer-statistics/statistics-by-cancer-type/bowel-cancer

2. Giovannucci E WK Cotcar ISD, Fraumeni J, eds. Cancer. Epidemiology and Prevention. 3rd ed. Oxford University Press (2006).

3. Turk PS and Wanebo HJ. "Results of surgical treatment of nonhepatic recurrence of colorectal carcinoma". Cancer 71 (1993): 4267-4277.

4. Santiago RJ., et al. "Chemoradiotherapy in the treatment of rectal cancer". Hematology/Oncology Clinics of North America 16 (2002): 995-1014.

5. Garcia-Aguilar J., et al. "Optimal timing of surgery after chemoradiation for advanced rectal cancer: preliminary results of a multicenter, nonrandomized phase II prospective trial". Annals of Surgery 254 (2011): 97-102.

6. de Campos-Lobato LF., et al. "Neoadjuvant therapy for rectal cancer: the impact of longer interval between chemoradiation and surgery". Journal of Gastrointestinal Surgery 15 (2011): 444-450.

7. Richards $\mathrm{CH}$., et al. "The impact of perioperative risk, tumor pathology and surgical complications on disease recurrence following potentially curative resection of colorectal cancer". Annals of Surgery 254 (2011): 83-89.

8. West MA., et al. "The effects of neoadjuvant chemoradiotherapy on physical fitness and morbidity in rectal cancer surgery patients". European Journal of Surgical Oncology 40 (2014): 1421-1428.

9. Moran J., et al. "Role of cardiopulmonary exercise testing as a risk-assessment method in patients undergoing intra-abdominal surgery: a systematic review". British Journal of Anaesthesia 116 (2016): 177-191.

10. Jack S., et al. "The effect of neoadjuvant chemotherapy on physical fitness and survival in patients undergoing oesophagogastric cancer surgery". European Journal of Surgical Oncology 40 (2014): 1313-1320.

11. Schubert C., et al. "The association between fatigue and inflammatory marker levels in cancer patients: a quantitative review". Brain, Behaviour and Immunity 21 (2007): 413-427. 
12. "ATS/ACCP Statement on cardiopulmonary exercise testing". American Journal of Respiratory and Critical Care Medicine 167 (2003): 211-277.

13. Sarhill N., et al. "Assessment of nutritional status and fluid deficits in advanced cancer". American Journal of Hospice and Palliative Care 20 (2003): 465-473.

14. Roberts HC., et al. "A review of the measurement of grip strength in clinical and epidemiological studies: towards a standardised approach". Age and Ageing 40 (2011): 423-429.

15. Foster KR and Lukaski HC. "Whole-body impedance--what does it measure?" The American Journal of Clinical Nutrition 64 (1996): 388S-396S.

16. Kushner RF., et al. "Use of bioelectrical impedance analysis measurements in the clinical management of patients undergoing dialysis". The American Journal of Clinical Nutrition 64 (1996): 503S-509S.

17. Klampfer L. "Cytokines, inflammation and colon cancer". Current Cancer Drug Targets 11 (2011): 451-464.

18. Aldinucci D and Colombatti A. "The inflammatory chemokine CCL5 and cancer progression". Mediators of Inflammation (2014): 292376.

19. He XX., et al. "Macrophage migration inhibitory factor promotes colorectal cancer". Molecular Medicine (Cambridge, Mass) 15 (2009): 1-10.

20. Palma G., et al. "Interleukin 18: friend or foe in cancer". Biochimica et Biophysica Acta 1836 (2013): 296-303.

21. Waldner MJ., et al. "Interleukin-6--a key regulator of colorectal cancer development". International Journal of Biological Sciences 8 (2012): 1248-1253.

22. Lasek W., et al. "Interleukin 12: still a promising candidate for tumor immunotherapy?" Cancer Immunology, Immunotherapy 63 (2014): 419-35.

23. Leitch EF., et al. "Comparison of the prognostic value of selected markers of the systemic inflammatory response in patients with colorectal cancer". British Journal of Cancer 97 (2007): 1266-1270.

24. DeVita VT Jr and Chu E. "A history of cancer chemotherapy". Cancer Research 68 (2008): 8643-8653.

25. "Local recurrence rate in a randomised multicentre trial of preoperative radiotherapy compared with operation alone in resectable rectal carcinoma. Swedish Rectal Cancer Trial". European Journal of Surgery 162 (1996): 397-402.
26. Ruo L and Guillem JG. "Major 20th-century advancements in the management of rectal cancer". Diseases of the Colon and Rectum 42 (1999): 563-578.

27. Skarlatos J., et al. "Hypofractionated radiotherapy with concurrent 5-fluorouracil radiosensitisation for recurrent or locally advanced colorectal cancer. A phase II study". International Journal of Colorectal Disease 11 (1996): 206-210.

28. Guren MG., et al. "Dietary intake and nutritional indicators are transiently compromised during radiotherapy for rectal cancer". European Journal of Clinical Nutrition 60 (2006): 113119.

29. Tsujimoto H., et al. "Preoperative chemoradiotherapy for esophageal cancer enhances the postoperative systemic inflammatory response". Japanese Journal of Clinical Oncology 36 (2006): 632-637.

30. Wilson RJ., et al. "Impaired functional capacity is associated with all-cause mortality after major elective intra-abdominal surgery". British Journal of Anaesthesia 105 (2010): 297-303.

31. Snowden CP., et al. "Submaximal cardiopulmonary exercise testing predicts complications and hospital length of stay in patients undergoing major elective surgery". Annals of Surgery 251 (2010): 535-541.

32. Ellis KJ. "Human body composition: in vivo methods". Physiology Review 80 (2000): 649-680.

33. Lopes JP., et al. "Nutritional status assessment in colorectal cancer patients". Nutrición Hospitalaria 28: 412-418.

34. Ollenschlager G. "Nutritional deficiency during aggressive treatment of tumors". Fortschr Med 109 (1991): 533-4

35. de Luis DA., et al. "Postsurgery enteral nutrition in head and neck cancer patients". European Journal of Clinical Nutrition 56 (2002): 1126-1129.

36. Cosnes J., et al. "Malnutrition in chronic radiation enteritis. Study of 100 patients". Annals of Clinical Gastroenterology and Hepatology 24 (1988): 7-12.

37. Deitel M and To TB. "Major intestinal complications of radiotherapy. Management and nutrition". Archives of Surgery 122 (1987): 1421-1424.

38. Cohen S., et al. "Muscle wasting in disease: molecular mechanisms and promising therapies". Nature Reviews Drug Discovery 14 (2015): 58-74. 
39. Gupta D., et al. "Malnutrition was associated with poor quality of life in colorectal cancer: a retrospective analysis". Journal of Clinical Epidemiology 59 (2006): 704-709.

40. West MA., et al. "The effect of neoadjuvant chemoradiotherapy on whole-body physical fitness and skeletal muscle mitochondrial oxidative phosphorylation in vivo in locally advanced rectal cancer patients--an observational pilot study". PLoS One 9 (2014): e111526.

41. Deboeck G., et al. "Exercise to predict outcome in idiopathic vs associated pulmonary arterial hypertension". European Respiratory Journal 40 (2012): 1410-1419.

42. Kosmas C., et al. "Cardiotoxicity of fluoropyrimidines in different schedules of administration: a prospective study". Journal of Cancer Research and Clinical Oncology 134 (2008): 75-82.

43. Tsibiribi P., et al. "Cardiac lesions induced by 5-fluorouracil in the rabbit". Human and Experimental Toxicology 25 (2006): 305-309.

\section{Assets from publication with us}

- Prompt Acknowledgement after receiving the article

- Thorough Double blinded peer review

- Rapid Publication

- Issue of Publication Certificate

- High visibility of your Published work

Website: www.actascientific.com/

Submit Article: www.actascientific.com/submission.php

Email us: editor@actascientific.com

Contact us: +919182824667 